\title{
NEUROPHARMACOLOGICAL EFFECTS OF THE ETHANOLIC EXTRACT OF DERIVATIVES OF LUPEOL IN RATS
}

\author{
ANKITA WAL*, PRANAY WAL, RUCHI TIWARI \\ Department of Pharmacy, Pranveer Singh Institute of Technology, Kanpur, Uttar Pradesh, India. Email: walankita@gmail.com
}

Received: 17 July 2018, Revised and Accepted: 18 September 2018

\section{ABSTRACT}

Objective: The neuropharmacological activities of ethanolic extract derivatives of lupeol are being screened on rats. Prepared derivatives are evaluated for their locomotor, anxiolytic and stereotype activities. The present investigation was designed to evaluate the antipsychotic effect of semisynthetic derivatives of lupeol using rat models of elevated maze model and apomorphine-induced stereotype behavior.

Methods: Lupeol was extracted from Crataeva nurvala bark using ethano. After chemical modification, we made different derivatives using aldol condensation. Different derivatives were obtained from a series of reaction previously published LAH-3, LAP-3, LAPEA-3, LAMP-3, LATS-3, and LAS-3. Neuropharmacological effects, including anxiolytic, central nervous inhibitory, and stereotype antipsychotic effects were evaluated in the different derivatives of lupeol at a dose of $250 \mathrm{mg} / \mathrm{kg}$ using standard methods.

Results: The absolute derivatives of LAH3 and LAPEA3 showed a significant reduction in the activity score in actophotometer test. Reduction in the locomotor activity indicates central nervous system (CNS) depressant property of the drug. LAMP3 and LAS3 show a significant anxiolytic effect. From the result of elevated plus maze, it was evident that derivatives of lupeol treated animals exhibit an increased number of entries into open arm when compared to normal control, which shows the anxiolytic activity of the lupeol derivatives. Sniffing, rearing and licking activities for lupeol derivatives LAH3 and LAPEA3 were found to be 35\%, 33\%, and 40\% and 40.33\%, 38\%, and 33.33\% respectively when it compared with standard and control groups. This model is suggestive of the absence of negative symptoms alleviating property of all the treatment groups.

Conclusions: The lupeol and its semisynthetic derivatives possess anxiolytic, CNS inhibitory, and antipsychotic effects to varying degree.

Keywords: Lupeol, Apomorphine, Anxiolytic, Antipsychotic.

(C) 2019 The Authors. Published by Innovare Academic Sciences Pvt Ltd. This is an open access article under the CC BY license (http://creativecommons. org/licenses/by/4. 0/) DOI: http://dx.doi.org/10.22159/ajpcr.2019.v12i1.28533

\section{INTRODUCTION}

The Indian system of medicines, namely, Ayurveda, Siddha, Unani, and Homeopathic system predominantly use plant-based raw materials and most of their preparations and formulations. Herbal medicines are becoming more and more popular nowadays [1]. Nowadays use of herbal medicinal products and supplements has increased tremendously over the past three decades which shows its immense potential towards healing diseases. Triterpenoids abundantly exist in the plant kingdom and largely derived from vegetable oils, cereals, and fruits [2]. Since ancient times, they have been used as remedies to treat human diseases. During the past decade, there has been an unprecedented increase in research on triterpenoids and focused on the anticancer and antidiabetic properties of triterpenoids. The past decade has also witnessed an intense interest in herbal medicines that have long-term health-promoting qualities. Herbal remedies for neurodegenerative disease is becoming more popular in the recent years as they show the possibility to slow down the brain's degeneration [3]. Triterpenoids isolated from Crataeva nurvala (Capparidaceae) displayed a broad spectrum of biological activities. Pentacyclic lupane-type triterpenes, exemplified by lupeol [lup-20(29)-en-3b-ol], principally found in common fruit plants such as olive, mango, strawberry, and grapes were reported to possess beneficial effects as a therapeutic and preventive agent for a range of disorders. It is noteworthy that lupeol at its effective therapeutic doses exhibits no toxicity to normal cells and tissues. Lupeol isolated from the leaves of Aegle marmelos appeared to be a good drug for antidyslipidemia and antihyperglycemia [2] and even hypercholesterolemia [3]. Lupeol is a diet-derived agent (acceptable to humans), nontoxic, and bioavailable, and a dose-tailored treatment was successful in the treatment of prostate cancer. Among these classes of compounds, the pentacyclic triterpene such as Lupeol, $\beta$ sitosterol, and lupenone is obtained from the stem bark of $C$. nurvala bark and, from many other plants, is particular interest because of its wide spectrum of biologically activity exhibited by it given in various literature [4], such as antiarthritis, antitumour, anti-inflammatory, hepatoprotective, antimalarial, and against free radical-induced toxicity, and their various semi-synthetic derivatives posses either higher activity or different activity. Root bark is preferred part of plant because of its potential pharmacological activity. It is usually harvested, sun-dried, and pulverized to obtain a powder. Lupeol isolated from Crataeva nurvala using 95\% ethanol. Then, the extract was concentrated on rotator evaporator under pressure which on repeated crystallization yielded pure lupeol. Further amount of lupeol was isolated by flash column chromatography of mother liquor along with small amount of lupenone. Previously, investigations in our laboratory revealed that lupeol, and their semisynthetic derivatives were active against antimalarial and tuberculosis $[5,6]$. These activities support the traditional notion of the use of this plant.

In this study, possible neuropharmaological effect of lupeol and their different using rats. The results will provide pharmacology data of lupeol and their derivatives.

\section{METHODS}

The bark of Crataeva was collected from local market. Stem bark of C. nurvala $(80 \mathrm{~kg}$ ) was authenticated from Chandra Shekhar Azad, University of Agriculture and Technology, Kanpur, and its reference is CSA/DOH/2016-2017/31 for this study. The root bark was separated and cleaned, then sun-dried, and pulverized using a mechanical grinder. It was coarsely powdered and extracted with 95\% ethanol. Crude lupeol obtained from concentrated ethanol extract was filtered out and crystallized with chloroform. The filtrate and collective mother 
liquor from crystallization were chromatographed over normal silica gel and packed in hexane and elution with while elution with 7-10\% ethyl acetate in hexane yielded lupeol. Now, we have prepared different derivatives of lupeol through aldol condensation, first, lupeol was converted into lupeol aldehyde, and then, lupeol aldehyde was reacted with different aliphatic and aromatic amine [7].

\section{Drugs}

Diazepam, apomorphine hydrochloride, diazepam (Sigma Chemical Co., USA), dimethyl sulfoxide (DMSO), and sodium chloride (Fisher Scientific Co. USA) were used.

\section{Animals}

Wistar albino rats (150-170 g) were maintained at the Animal Facility Center, PSIT, under standard environmental condition of temperature $\left(22 \pm 3^{\circ} \mathrm{C}\right)$, relative humidity $(14 \pm 1)$ and light/dark cycles $(12 / 12 \mathrm{~h})$. The animals were fed with grains and water ad libitum. The protocol of all the experiments was approved by the Institutional Ethical Committee (Approval number 1273/PO/Re/S/09/CPSEA).

\section{Pharmacological evaluation}

For locomotary behavior, actophotometer was used for screening antipsychosis in animal, while rota-rod was used for evaluation of neurological depression activity. Locomotor activity indicates decrease in fall off time which suggests depression in central nervous system (CNS) [8].

\section{Procedure}

Six animals were used in each group for each experiment separately. Animals treated with DMSO (0.8 ml p.o.) served as control. Diazepam ( $2 \mathrm{mg} / \mathrm{kg}$ i.p.) served as standards, and animals in the test group were treated with different derivatives of lupeol $(250 \mathrm{mg} / \mathrm{kg}$ i.p.), respectively. Each animal was treated with respective drug $30 \mathrm{~min}$ before the experiment. Tests performed were as follows. The rats were numbered and weighted. The semi-synthesized derivatives of lupeol such as LAH-3, LAP-3, LAPEA-3, LAMP-3, LATS-3, and LAS-3 were selected for evaluated for their anxiety responses using actophotometer. The actophotometer was switched on, and each rat was placed in the actophotometer for $10 \mathrm{~min}$. The basal locomotor activity score of all rats was noted. Test and control samples were injected to proposed group of rats. Each animal was retested for $10 \mathrm{~min}$.

\section{Elevated plus maze (EPM) model}

This model represented behavioral activities of rat after administration of lupeol derivatives and the activities were observed by the person unaware of the treatment of rats [9]. Animals were housed at four per cage, allowed free access to water and food, and maintained under constant temperature $\left(23 \pm 1^{\circ} \mathrm{C}\right)$ and humidity $(60 \pm 10 \%)$ under a 12 -h light/dark cycle (light on 07.30-19.30 h). The ratio of open and closed place entries showed a particular effect on anxiety when there is no prominent change in the total number of open and closed entries. The behavioral alteration patterns shown by derivatives of lupeol in this EPM model reflect the anxiolytic effect as given in Table 1. Six animals were used in each group for each experiment separately. Animals were treated with distilled water $(0.65 \mathrm{ml}$ p.o. $)$ served as control. Diazepam $(2 \mathrm{mg} / \mathrm{kg}$ i.p.) served as standards, and animals in the test group were treated with different derivatives of lupeol ( $250 \mathrm{mg} / \mathrm{kg}$ i.p.), respectively. Each animal was treated with the respective drug $30 \mathrm{~min}$ before experiment.

\section{Apomorphine stereotypy in rats}

The stereotype is frequent mechanical repetition of a movement. It is an isolated motor acts or partial sequence of more complex behavioral patterns from the repertoire of the species, occurring out of the context and with an abnormally high frequency [10].

Wistar albino rat (100-50 g) was obtained from the laboratory animal center. The rat was housed in plastic cages in a group of six animals under standard laboratory conditions at temperature of $22 \pm 1^{\circ} \mathrm{C}$ and $12 \mathrm{~h}$

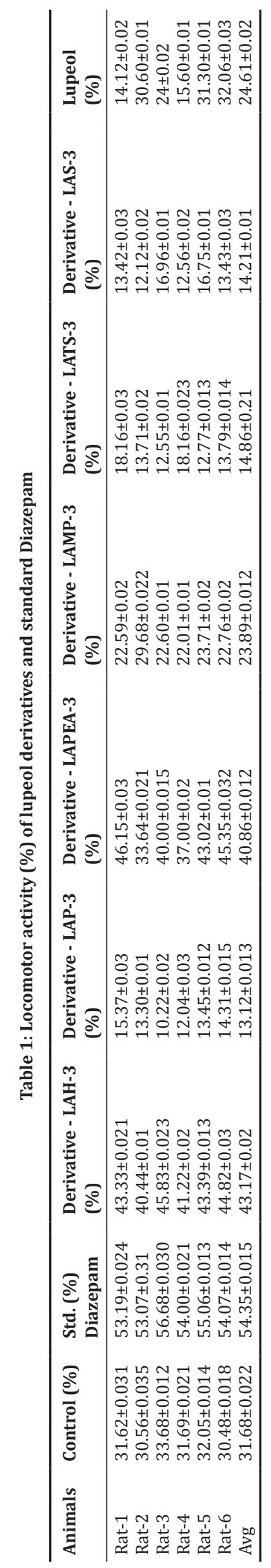


light:12 h dark cycle. Animals were fed with standard laboratory, food pellet, and water ad libitum. The animals were acclimatized to the experimental room and handled for 1 week before start of the experiment. Six animals were used in each group for each experiment separately. Animals treated with distilled water $(0.65 \mathrm{ml}$ p.o.) served as control. Apomorphine $\mathrm{HCl}$ $(1.5 \mathrm{mg} / \mathrm{kg}$ body weight, ip) served as standards, and animals in test group were treated with different derivatives of lupeol $(250 \mathrm{mg} / \mathrm{kg}$ i.p.), respectively. Each animal was treated with respective drug $30 \mathrm{~min}$ before the experiment. All the drug solutions were prepared fresh in distilled water and administered intraperitoneally (i.p.) in a constant volume of $1 \mathrm{ml} / 100 \mathrm{~g}$ body weight of the animal. Apomorphine hydrochloride was dissolved in distilled water. A $10 \mathrm{~s}$ observation period was used for measuring the presence of stereotype activity such as sniffing, licking, and chewing $10 \mathrm{~min}$ after apomorphine injection. The percent effectiveness of drug was estimated by a number of animals protected in each group [11].

\section{Statistical analysis}

The results were expressed as mean \pm standard error of the mean (SEM). Data were analyzed by ANOVA followed by turkey's test. $p<0.05$ was taken to be statistically significant. The statistical analysis was done using the software SPSS. Results were presented as Figs. 1-7.

\section{RESULT}

The plant Crateva nurvala containing triterpenoid was identified as lupeol [12]. Our interest is to prepare semisynthetic derivatives of lupeol by chemical transformation and evaluate their pshychopharmacological activities.

\section{Anxiolytic response}

The reduction in the entries and time spent in open arms indicate a high level of fear or anxiety. The decrease in aversion to open arms is the result of anxiolytic effect, expressed by the time spent, and entries in the open arm [13]. Anxiolytic agents increase the motor activity, which manifests an increase in the time spent by the animal in the open arms of EPM [14]. Diazepam was taken as a control hat dose of $2 \mathrm{mg} /$ kg orally; it was statistically significant $(\mathrm{p}<0.01)$ CNS depressant and muscle relaxant activity. LAH 3 and LAPEA3 here showed significant $(p<0.05)$ progressive decrease in the time of fall from the rotarod. Lupeol derivatives LAH3 and LAPEA3 revealed significant reduction in activity score during actophotometer test. Reduction in the locomotor activity indicates CNS depressant property of the drug. The percentage change in locomotor activity of vehicle-treated animal is $5.5 \%$, while the lupeol derivative shows 10 times more activity (43.20\% and 39.93\%) than the control and standard drug diazepam shows $31.95 \%$ and $54.31 \%$ reduction in locomotor activity. Effect of lupeol derivatives on animal behavior was observed by actophotometer. Compound LAH3 and LAPEA3 showed significant difference with $\mathrm{p}<0.05$. Diazepam $(2 \mathrm{mg} / \mathrm{kg}$, oral) and drug derivatives $(250 \mathrm{mg} / \mathrm{kg}$, oral) treated groups showed significant locomotor activity when compared with control; however; this anxiolytic activity was less with drug derivatives treated group than diazepam-treated group. Values expressed as mean \pm SEM, $\mathrm{n}=6$ in each group.

\section{EPM model}

The behavioral alteration patterns shown by derivatives of lupeol in this EPM model reflect the anxiolytic effect as given in Fig. 1.

The behavioral alterations induced by the lupeol and its derivatives CSA/DOH/2016-2017/31 for this study. The root bark was separated The behavioral alterations induced by the lupeol and its derivatives in the EPM significantly increased the arm entries in open arms and decreased the time spent and arm entries in the closed arms in a similar fashion; diazepam increased the time spent and arm entries in the open arms of lupeol, and its derivatives at a dose of $250 \mathrm{mg} / \mathrm{kg}$ significantly increased the entries in open and closed arm and decreased the time spent when it compared with control. In this study, the number of entries into open arm and time spent into open arm were taken, and

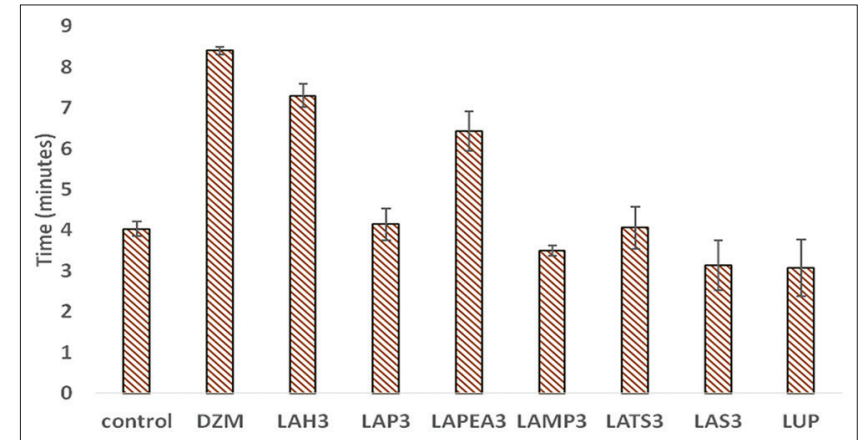

Fig. 1: Antianxiety activity of derivatives of lupeol on rat using elevated plus maze model time spent with derivatives

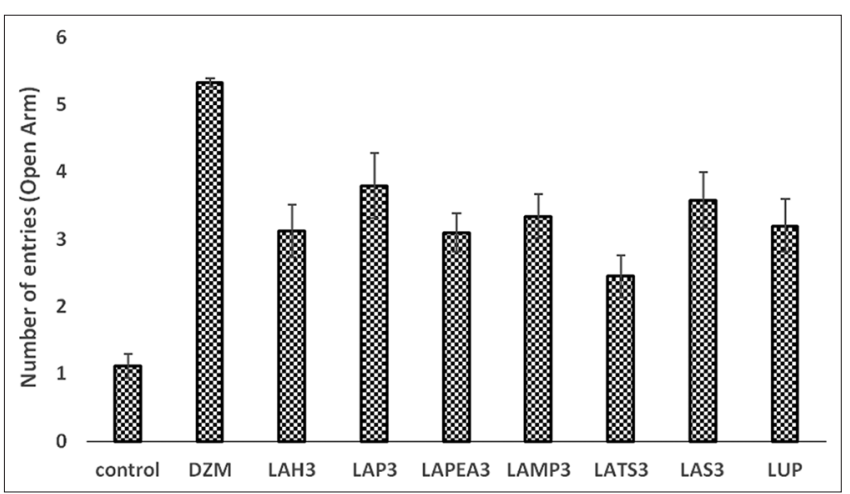

Fig. 2: Number of entries (open arm) with derivatives

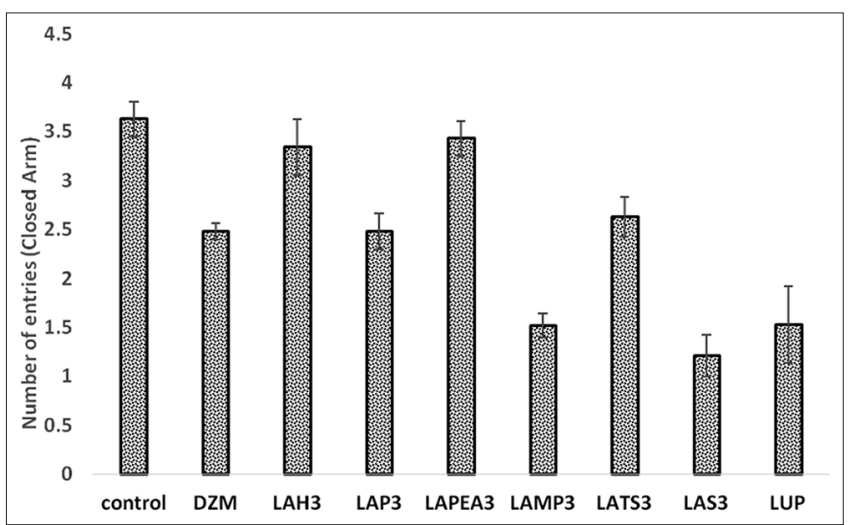

Fig. 3: Number of entries (closed arm) with derivatives

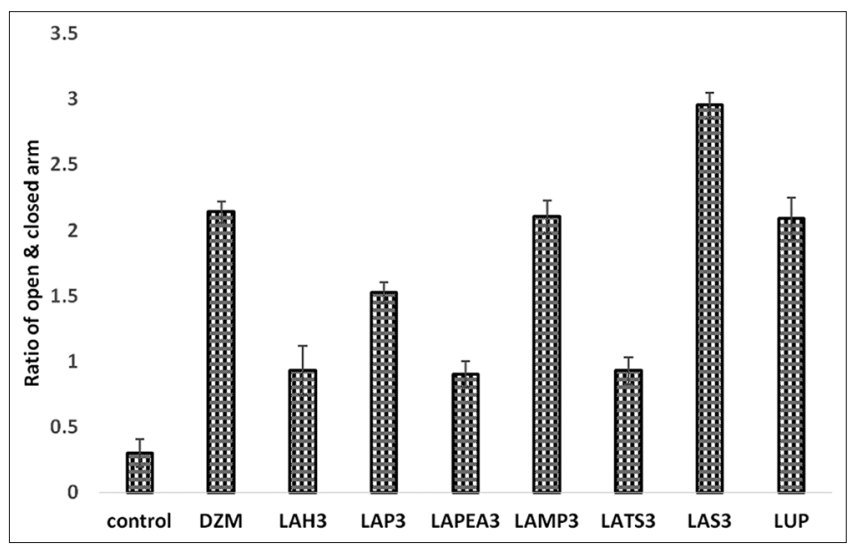

Fig. 4: Ratio of open and closed arm with derivatives 


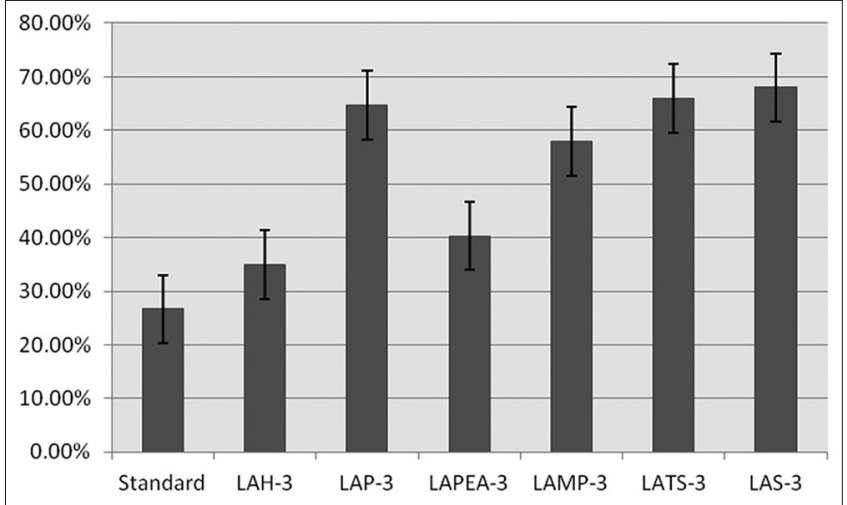

Fig. 5: Rearing behavior of animal the number of entries and time spent in different parts of maze were taken as a measurement of anxiety by EPM model.

\section{Apomorphine stereotypy in rats}

Apomorphine (1.5 mg/kg i.p.) and drug derivatives $(250 \mathrm{mg} / \mathrm{kg}$, oral) significantly $(\mathrm{p}<0.001)$ exhibited psychosis, as evident from decreased rearing, sniffing, and licking behaviour as compared with control (Figs. 5-7). Lupeol derivatives such as LAH3 and LAPEA3 have shown to exert a positive effect in animal models used in the search for putative antipsychotic-like drugs. The predictive validity of these animal models is based on a comparison of the test drug with a well-established antipsychotic drug such as apomorphine. Results from this study show that all the stereotypic activities such as sniffing, rearing, and licking were reduced significantly in all the treatment groups $(p<0.05)$ compared to the control groups, but the degree of reduction varied differently among the treatment groups with no significant difference

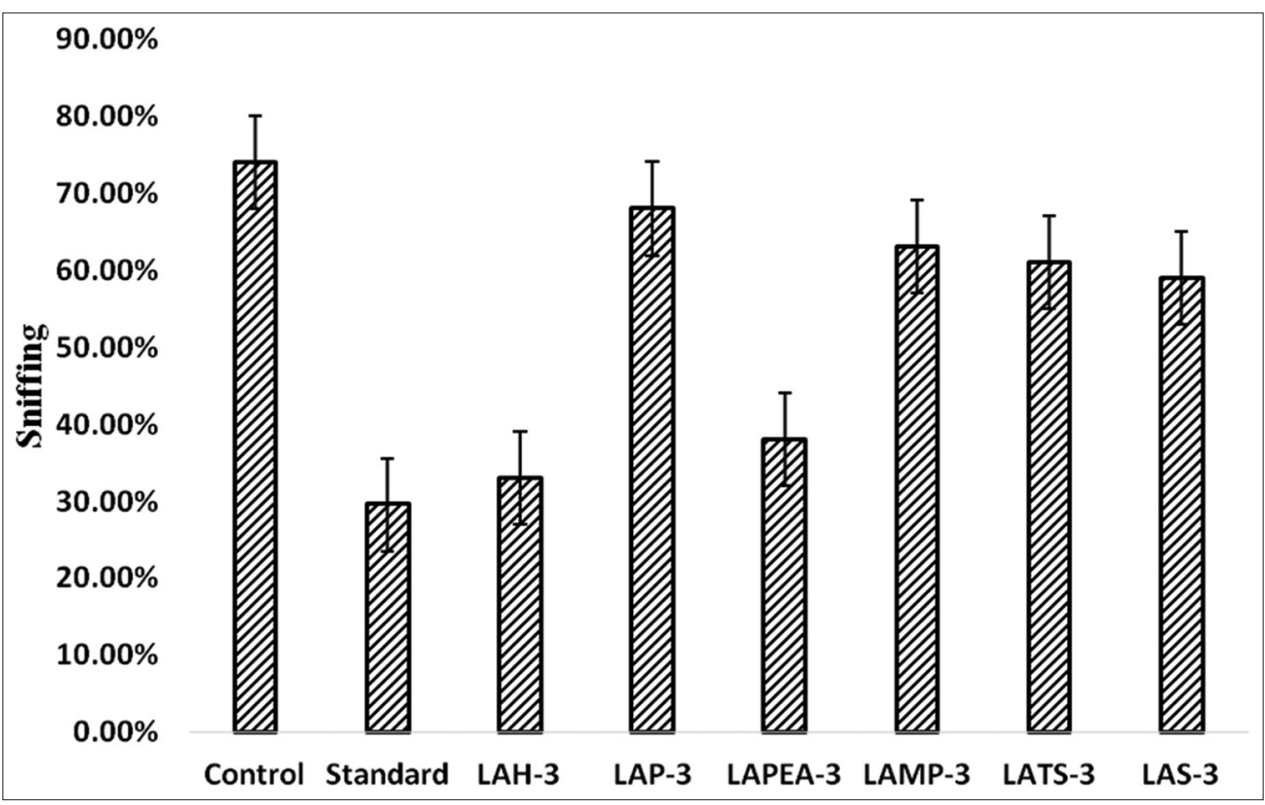

Fig. 6: Sniffing behavior of animal

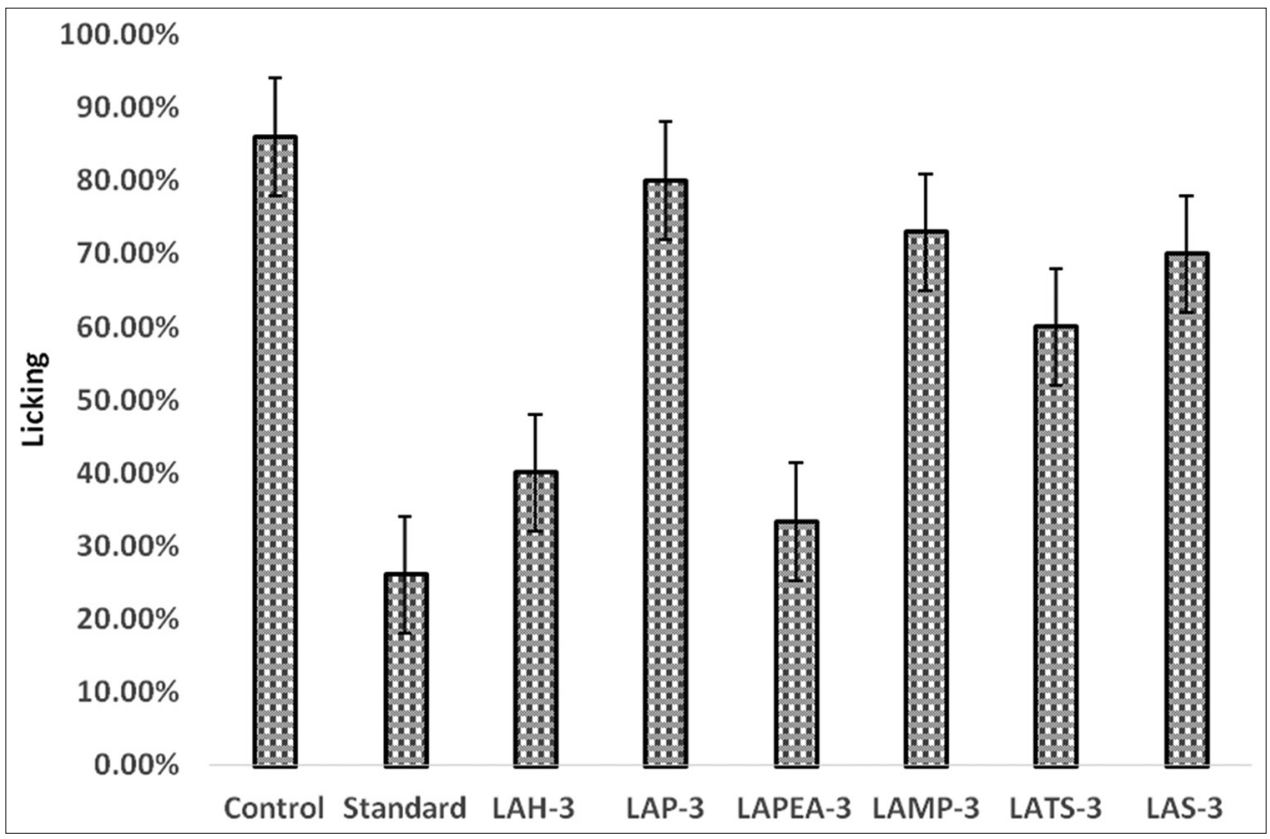

Fig. 7: Licking behavior of animal 
among the lupeol derivatives. The standard drug amphetamine reduced sniffing, rearing, and licking activity by $28.17 \%, 26.66 \%$, and $26 \%$, respectively. The lupeol derivatives such as LAH3 reduced sniffing, rearing, and licking activity by $35 \%, 33 \%$, and $40 \%$, respectively, whereas the LAPEA3 derivatives reduced sniffing, rearing, and licking by $40.33 \%, 38 \%$, and $33.33 \%$, respectively, when compared with standard and control groups. This model is suggestive of the absence of negative symptoms alleviating property of all the treatment groups.

\section{DISCUSSION}

In modern life, anxiety, insomnia, and psychosis are widely spread in modern fast-paced life with a multitude of stressful conditions. Present study stated that lupeol derivatives showed significant effect of antipsychotic activity in rats. Few of neuropharmacological activities were reported earlier. For anxiolytic response, anxiolytic agents increase the motor activity, which manifests as an increase in the time spent with the animal in the open arms of EPM. LAH 3 and LAPEA3 here showed significant $(\mathrm{p}<0.05)$ progressive decrease in the time of fall from the rotarod. The absolute derivatives of LAH3 and LAPEA3 showed a significant reduction in the activity score in actophotometer test. Reduction in the locomotor activity indicates CNS depressant property of the drug. Derivative of lupeol LAMP3 and LAS3 shows a significant anxiolytic effect. From the result of EPM, it was evident that derivatives of lupeol treated animals exhibit an increased number of entries into open arm when compared to normal control, which shows the anxiolytic activity of lupeol.

For stereotype behavior, lupeol derivatives such as LAH3 reduced sniffing, rearing, and licking activity by $35 \%, 33 \%$, and $40 \%$, respectively, whereas the LAPEA3 derivatives reduced sniffing, rearing, and licking by $40.33 \%, 38 \%$, and $33.33 \%$, respectively, when compared with a standard and control groups.

\section{CONCLUSION}

The natural products include compounds from plants, microbes, animals, and synthetic or semi-synthetic compounds based on natural drugs. They have been used to treat a variety of disease conditions such as cancer, infections, diabetic, and other metabolic diseases. From the result obtained, we concluded that derivatives of lupeol such as LAH3 and LAPEA3 possess anxiolytic and antipsychotic activity. The predictive validity of these animal models is based on a comparison of the test drug with a well-established antipsychotic drug such as apomorphine. All the pharmacological studies were presented depicting the clear indications of the inferences drawn.

\section{AUTHORS' CONTRIBUTIONS}

Dr. Ankita Wal has worked in the extraction and isolation of lupeol, formed their derivatives, and done all the animal models. Dr. Pranay wal helped in pharmacological screening in animal modelling. Dr. Ruchi Tiwari takes the responsibility to prepare graphs in the manuscript.

\section{CONFLICTS OF INTEREST}

The authors declare that there are no conflicts of interest concerning the content of this article.

\section{REFERENCES}

1. Kalidhar SB, Meera G. Chemical constituents of Crateava nurvala (buch-ham) leaves. Indian J Pharm Sci 2006;68:804-6.

2. Siddique HR, Mishra SK, Karnes RJ, Saleem M. Lupeol, a novel androgen receptor inhibitor: Implications in prostate cancer therapy. Clin Cancer Res 2011;17:5379-91.

3. Kweifio-Okai G, De Munk F, Macrides TA, Smith P, Rumble BA. Antiarthritic mechanisms of lupeol triterpenes. Drug Dev Res 1995;36:20-4

4. Vogel HG. Drug Discovery and Evaluation: Pharmacological Assays. $3^{\text {rd }}$ ed. New York: Springer-Verlag Berlin Heidelberg; 2008. p. 1103.

5. Tiwari DK, Nagar H, Dwivedi G, Tripathi RK, Jena J. Evaluation of anti-anxiety activity of Plectranthus amboinicus (Lour.) on rats. Asian J Pharm Clin Res 2012;5 Suppl 4:110-3.

6. Parmar NS, Prakash S. Screening Methods in Pharmacology. New Delhi: Narosa Publishing House; 2013.

7. Wal A, Srivastava RS, Rai AK, Wal P, Tiwari R. Synthesis and evaluation of antipsychotic compounds from lupeol. Pharm Methods 2017;8:81-6.

8. Wal A, Srivastava RS, Rai AK. Synthesis and antitubercular activity of novel lupeol derivatives. Pharm Lett 2016;8:79-85.

9. Sukhdev, editor. Handbook of Terpenoids. Vol. 1. Florida, USA: CRC Press; 1989.

10. Mason G, Rushen J. Stereotypic Animal Behavior: Fundamentals and Applications to Welfare. $2^{\text {nd }}$ ed. Wallingford: CABI; 2006. p. 1-15.

11. Badyal DK, Desai C. Animal use in pharmacology education and research: The changing scenario. Indian J Pharmacol 2014;46:257-65.

12. Sudhakar P, Gopalakrishna HN, Swati B, Shreyasi C, Vinod N. Antianxiety effect of aqueous extract of fruits of Emblica officinalis(EO) on acute and chronic administration in rats. J Pharm Res 2010;3:219-23.

13. Roy S, Awasthi H. Herbal medicines as neuroprotective agent: A mechanistic approach. Int J Pharm Pharm Sci 2017;9:1-7.

14. Mangal A, Jain V, Jat RC, Bharadwaj S, Jain S. Neuro pharmacological study of leaves of Camellia sinensis. Int $\mathrm{J}$ Pharm Pharm Sci 2010;2 Suppl 3:132-34. 